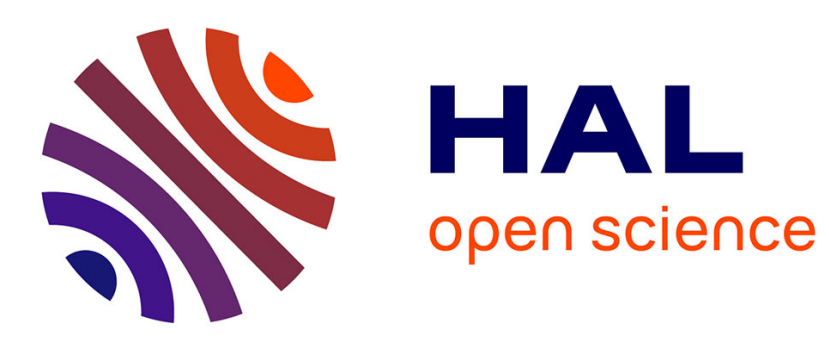

\title{
Confidence-Driven Control of an Ultrasound Probe: Target-Specific Acoustic Window Optimization
}

Pierre Chatelain, Alexandre Krupa, Nassir Navab

\section{To cite this version:}

Pierre Chatelain, Alexandre Krupa, Nassir Navab. Confidence-Driven Control of an Ultrasound Probe: Target-Specific Acoustic Window Optimization. IEEE Int. Conf. on Robotics and Automation, ICRA'16, May 2016, Stockholm, Sweden. hal-01274748

\section{HAL Id: hal-01274748 \\ https://inria.hal.science/hal-01274748}

Submitted on 16 Feb 2016

HAL is a multi-disciplinary open access archive for the deposit and dissemination of scientific research documents, whether they are published or not. The documents may come from teaching and research institutions in France or abroad, or from public or private research centers.
L'archive ouverte pluridisciplinaire HAL, est destinée au dépôt et à la diffusion de documents scientifiques de niveau recherche, publiés ou non, émanant des établissements d'enseignement et de recherche français ou étrangers, des laboratoires publics ou privés. 


\title{
Confidence-Driven Control of an Ultrasound Probe: Target-Specific Acoustic Window Optimization
}

\author{
Pierre Chatelain ${ }^{1}$, Alexandre Krupa ${ }^{2}$ and Nassir $\mathrm{Navab}^{3}$
}

\begin{abstract}
We propose a control framework to optimize the quality of robotic ultrasound imaging while tracking an anatomical target. We use a multitask approach to control the in-plane motion of a convex probe mounted on the end-effector of a robotic arm, based not only on the position of the target in the image, but also on features extracted from an ultrasound confidence map. The resulting control law therefore guarantees a good image quality, while keeping the target aligned with the central ultrasound scan-line. Potential applications of the proposed approach are, for example, teleoperated ultrasound examination, motion compensation for ultrasound-guided interventions, or automatic ultrasound acquisition. We demonstrate our approach with experiments on an ultrasound examination training phantom in motion.
\end{abstract}

\section{INTRODUCTION}

Ultrasound is an attractive modality for both diagnostic and interventional imaging, because it is non-invasive, low cost, and real-time. The quality of ultrasound images depends on intrinsic factors, such as the hardware design and the different acquisition parameters, but also on the conditions of the examination. Indeed, the placement of the probe, the ultrasound beam incidence angle, the contact force applied to the body, and the distribution of ultrasound gel are critical factors to obtain a good image quality. Finding the optimal acoustic window to observe the anatomy of interest requires expertise, especially for locations where dense objects, such as bones, generate shadows in the image. The quality of ultrasound images has been greatly improved by more and more efficient hardware design in the past decades. The use of ultrasound contrast agents to increase the contrast of ultrasound images has also been studied as a way to improve the image quality [1]. Post-processing algorithms, such as denoising or speckle reduction, can also enhance the acquired images [2], [3]. For a detailed overview of ultrasound image enhancement, the reader is referred to [4].

However, even with a modern transducer design and improved post-processing techniques, the quality of ultrasound images remains dependent on the expertise of the clinician. This issue has motivated the development of robot-assisted ultrasound systems, and of tele-echography in particular, in order to enable remote examination by specialists of patients

\footnotetext{
${ }^{1}$ Pierre Chatelain is with Université de Rennes 1, IRISA, France and Computer Aided Medical Procedures, Technische Universität München, Germany pierre. chatelaindirisa.fr

${ }^{2}$ Alexandre Krupa is with Inria Rennes - Bretagne Atlantique, IRISA, France alexandre. krupa@inria. fr

${ }^{3}$ Nassir Navab is with Computer Aided Medical Procedures, Technische Universität München, Germany and the Johns Hopkins University, MD, USA nassir.navabein.tum. de
}

located in medically isolated sites. While the first teleultrasound examinations where performed without robotic assistance, using a video conferencing system to guide a technician on site [5], robotic tele-echography systems started to be developped in the early 2000s [6]-[8]. In such systems, force sensors are used to ensure that the contact forces between the probe and the patient are not excessive, and, thanks to haptic devices, to provide a force feedback to the master controller [6], [8], [9].

Image control has also been of interest to facilitate the manipulation of the probe. In [7], Salcudean et al. use an ultrasound image correlation algorithm to track the carotid artery, and propose a shared control over the motion of the ultrasound probe. In [10], the out-of-plane translation of the carotid artery is controlled using an intensity-based method. In [11], intensity-based visual servoing of $2 \mathrm{D}$ and $3 \mathrm{D}$ probes is used to compensate for organ motion. A shared control method to maintain visibility constraints during teleechography is proposed in [12].

On the other hand, the impact of the probe placement on the image quality has received little attention up to now. Recently, Kuhlemann et al. [13] have proposed a robotic system to avoid shadowed areas based on entropy as a measure of quality. Entropy, and other statistical image analysis techniques [2], [14], are easy to estimate and can provide a first insight into the quality of ultrasound images. However, these methods do not account for the specificities of ultrasound. El-Zehiry et al. [15] proposed to learn a relation between the imaging parameters of the scanner and the image quality, based on expert ratings. The learned model was used to optimize the imaging parameters automatically. This framework provides an ultrasound-specific measure of quality, but the measure remains global for each image, and the position of the probe is not considered. Karamalis et al. introduced in [16] the concept of ultrasound confidence map, which provides a pixelwise measure of the ultrasound signal quality. The computation of Karamalis' confidence maps is based on a simplified physical model of sound propagation in soft tissues. Therefore, it provides an objective measure of quality. This framework has been found useful for shadow detection, to improve ultrasound reconstruction [16] or ultrasound image compounding [17], or as a visualization tool for the clinician [18]. In [19], we proposed to use the ultrasound confidence map as a new signal for servo-control, and designed a confidence-driven visual servoing control law to optimize the orientation of the probe with respect to the image quality. However, the quality-based control was global, without considering the anatomical contents of the image. In 
a clinical scenario, one would be interested in finding the best acoustic window for a specific target.

In this paper, we extend the method presented in [19] to address the tracking of a specific anatomical target. Our objective is thus (i) to maintain the target horizontally centered in the ultrasound image and (ii) to orientate the probe in order to get the best acoustic window for this target. We formalize these tasks, together with the force control task, using the redundancy framework [20] to ensure that the quality optimization task does not disturb the motion compensation task. The resulting framework provides a decoupled control of contact force, target positioning in the ultrasound image, and quality on the three in-plane degrees of freedom (DOF). The main contributions of this paper are thus:

- The definition of a quality optimization task with respect to a specific anatomical target, instead of the global image as was done in [19].

- The formalization of the control law with the redundancy framework.

In addition, we validate our method experimentally with an abdominal ultrasound training phantom.

We describe our control framework in Sect. II, starting with a short description of the confidence maps (Sect. II-A), and we present the results of experiments performed on a realistic ultrasound phantom in Sect. III. Finally, we propose a discussion of our method in Sect. IV.

\section{METHODS}

\section{A. Ultrasound Confidence maps}

Ultrasound confidence maps [16] provide a per-pixel measure of the ultrasound image quality. The confidence estimation is based on a graphical model of the physics of ultrasound image formation, with two boundary conditions: the confidence is 1 next to the transducer (perfect signal), and 0 at the maximal depth (no signal). This system of constraints is solved using the random walks algorithm [21]. The solution is a probabilistic map $C: \Omega \longrightarrow[0,1]$ over the field of view $\Omega$ where, for each pixel $(x, y) \in \Omega, C(x, y)$ is the confidence of the signal at this location.

\section{B. Control framework}

We consider a convex ultrasound probe attached to the end-effector of a 6-DOF robotic arm. Our goal is to optimize the visual quality of a given anatomical target. To this end, we define three functional tasks to be realized jointly:

1) Maintain a constant contact force between the probe and the patient.

2) Maintain the target horizontally centered in the image.

3) Optimize the confidence of the ultrasound signal at the target location.

We propose to use the redundancy formalism [20] to design a control law fulfilling the constraints defined above. We realize the fusion of the tasks in the probe frame $\mathcal{F}_{p}$ attached to the imaging center of the convex probe. We define

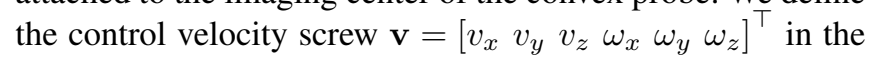
probe frame, with $v_{x}, v_{y}, v_{z}$ the translational velocities, and

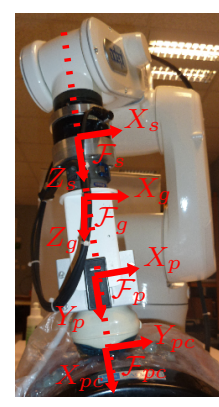

(a)

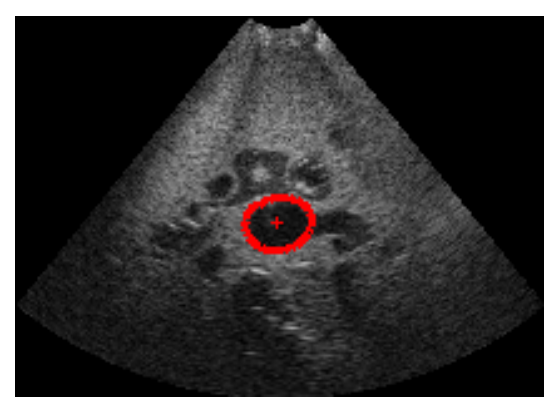

(b)
Fig. 1. (a) Robotic system and the corresponding force sensor frame $\left(\mathcal{F}_{s}\right)$, probe frame $\left(\mathcal{F}_{p}\right)$, probe contact frame $\left(\mathcal{F}_{p c}\right)$, and gravity frame $\left(\mathcal{F}_{g}\right)$. (b) An ultrasound image. The red line corresponds to the contour of the target. The red cross corresponds to the barycenter of the target.

$\omega_{x}, \omega_{y}, \omega_{z}$ the rotational velocities. The different frames involved in the design of our control law are represented in Fig. 1. The force sensor frame $\mathcal{F}_{s}$ is attached to a 6axes force/torque sensor mounted on the robotic arm, and the probe contact frame $\mathcal{F}_{p c}$ to the contact point between the probe and the object's surface. The probe gravity frame $\mathcal{F}_{g}$ is centered on the mass center of the probe, and its $z$-axis is aligned with the direction of the gravity force.

1) Force control: We start by describing the control law for the contact force task. We use the method proposed in [11], to which the reader may refer for further details. The force/torque sensor provides a measure of the force tensor ${ }^{s} \mathbf{H}_{s}$ expressed in the sensor frame $\mathcal{F}_{s}$ (see Fig. 1). In order to obtain the contact force between the probe and the patient, we also need to consider the action of gravity on the probe. The gravity force tensor expressed in the gravity frame $\mathcal{F}_{g}$ can be expressed as ${ }^{g} \mathbf{H}_{g}=\left[\begin{array}{lllllll}0 & 0 & 9.81 m_{p} & 0 & 0 & 0\end{array}\right]^{\top}$, where $m_{p}$ is the mass of the probe (in $\mathrm{kg}$ ). Given the twist transformation matrices ${ }^{p c} \mathbf{F}_{s}$ and ${ }^{s} \mathbf{F}_{g}$ allowing the transformation of a force/torque tensor from one frame to another, the force tensor in the contact frame $\mathcal{F}_{p c}$ reads:

$$
{ }^{p c} \mathbf{H}_{p c}={ }^{p c} \mathbf{F}_{s}\left({ }^{s} \mathbf{H}_{s}-{ }^{s} \mathbf{F}_{g}{ }^{g} \mathbf{H}_{g}\right) .
$$

As we are interested in controlling the force only along the $y$-axis of the probe, we define the force feature as the $y$ component of the force tensor: $s_{f}=\left[\begin{array}{llllll}0 & 1 & 0 & 0 & 0 & 0\end{array}\right]^{p c} \mathbf{H}_{p c}$. We denote $s_{f}^{*}$ the desired contact force, and $e_{f}=s_{f}-s_{f}^{*}$ the error to minimize. The aim of the contact force task is to obtain an exponential decrease of $e_{f}$ Therefore, the desired variation of the error is defined as $\dot{e}_{f}^{*}=-\lambda_{f} e_{f}$, where $\lambda_{f}$ is the force control gain. The interaction matrix $\mathbf{L}_{f}$ relating the variations of $s_{f}$ to the velocity screw $\mathbf{v}$ in the probe frame, such that $\dot{s}_{f}=\mathbf{L}_{f} \mathbf{v}$, can be approximated as

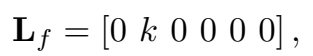

where $k$ is an estimation of the contact stiffness. Following the task function framework, the control law to apply in order to get the desired behavior can be written

$$
\mathbf{v}_{f}=\mathbf{L}_{f}^{+} \dot{e}_{f}^{*},
$$

where $L_{f}^{+}=\left[\begin{array}{llllll}0 & \frac{1}{k} & 0 & 0 & 0 & 0\end{array}\right]^{\top}$ is a generalized inverse of $L_{f}$. 


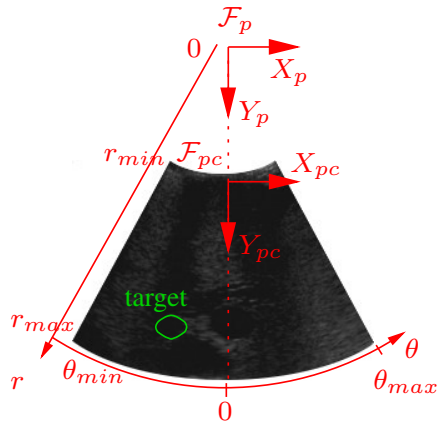

(a)

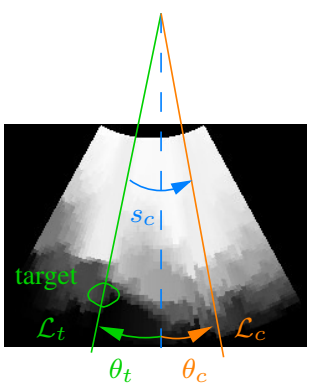

(b)
Fig. 2. Illustration of the different coordinate systems. (a) Probe frame and image polar coordinates. (b) Confidence map with the target angular coordinate $\theta_{t}$, the confidence main orientation $\theta_{c}$, and the confidence feature $s_{c}$.

2) Motion compensation: The target is tracked in the ultrasound images using the parametric active contour algorithm described in [22]. We denote $\left(x_{t}, y_{t}, z_{t}\right)$ the coordinates of the target's barycenter in the probe frame. Since we consider a $2 \mathrm{D}$ probe, the target is located in the image plane, so that $z_{t}=0$. The motion compensation task consists in maintaining the target centered horizontally in the image. Therefore, we define the visual feature $s_{t}=x_{t}$ and its desired value $s_{t}^{*}=0$. The desired error variation is $\dot{e}_{t}^{*}=-\lambda_{t}\left(s_{t}-s_{t}^{*}\right)$, where $\lambda_{t}$ is the control gain of the motion compensation task. The relation between the variation of the visual feature and the velocity screw $\mathbf{v}$ expressed in the probe frame is given by the interaction matrix $\mathbf{L}_{t}$ such that $\dot{s}_{t}=\mathbf{L}_{t} \mathbf{v}$ :

$$
\mathbf{L}_{t}=\left[\begin{array}{llllll}
-1 & 0 & 0 & 0 & 0 & y_{t}
\end{array}\right]
$$

3) Quality optimization: The quality task aims at optimizing the quality of the ultrasound image at the target location. To this end, a possible approach would be to search for the scan-line along which the signal confidence is maximized. However, the direction of maximum confidence would be an unstable feature, with possible temporal discontinuities. Instead, we use the angular coordinate $\theta_{c}$ of the confidenceweighted barycenter of the confidence map as an indicator of the best acoustic direction:

$$
\theta_{c}=\frac{1}{C_{\Omega}} \iint_{(r, \theta) \in \Omega} \theta C(r, \theta) r d r d \theta,
$$

where $C:\left[r_{\min }, r_{\max }\right] \times\left[\theta_{\min }, \theta_{\max }\right] \longrightarrow[0,1]$ is the confidence map, and $C_{\Omega}=\iint_{(r, \theta) \in \Omega} C r d r d \theta$. This measure and the corresponding scan-line $\mathcal{L}_{c}$ are represented in Fig. 2(b).

Our goal is to get the best image quality at the target location. Therefore, we define the confidence feature as the angular displacement $s_{c}=\theta_{c}-\theta_{t}$ between the target and the confidence barycenter, where $\theta_{t}=\operatorname{atan} \frac{x_{t}}{y_{t}}$ is the angular coordinate of the target. The angle $\theta_{t}$ corresponds to the scan-line $\mathcal{L}_{t}$ passing through the center of the target [see Fig. 2(b)]. As we wish to find the best acoustic window for the target, the desired configuration is $\mathcal{L}_{c}^{*}=\mathcal{L}_{t}^{*}$, corresponding to $s_{c}^{*}=0$. The desired variation of the confidence feature error is then defined as $\dot{e}_{c}^{*}=-\lambda_{c}\left(s_{c}-s_{c}^{*}\right)$, where $\lambda_{c}$ is the control gain of the quality optimization task. It is easy to show that

$$
\dot{\theta}_{t}=\left[\begin{array}{llllll}
\frac{\cos \theta_{t}}{r_{t}} & -\frac{\sin \theta_{t}}{r_{t}} & 0 & 0 & 0 & -1
\end{array}\right] \mathbf{v},
$$

where $r_{t}=\sqrt{x_{t}^{2}+y_{t}^{2}}$ is the radial coordinate of the target. According to [19], we can also write $\dot{\theta}_{c} \approx\left[\begin{array}{llllll}0 & 0 & 0 & 0 & 0 & -1\end{array}\right] \mathbf{v}$. Therefore, the interaction matrix $\mathbf{L}_{c}$ that relates the variations of $s_{c}=\theta_{c}-\theta_{t}$ to the velocity screw in the probe frame can be approximated as

$$
\mathbf{L}_{c}=\left[\begin{array}{llllll}
-\frac{\cos \theta_{t}}{r_{t}} & \frac{\sin \theta_{t}}{r_{t}} & 0 & 0 & 0 & 0
\end{array}\right] .
$$

4) Control fusion: The force control acts on the translation along $Y_{p}$, while the motion compensation acts on the translation along $X_{p}$ and the rotation around $Z_{p}$. As a result, these two controls are decoupled. On the other hand, it appears from (7) that the confidence control is coupled to the force and vision controls. In order to ensure that the confidence control does not disturb the other tasks, we use the redundancy framework to define the components $\mathbf{v}_{f}, \mathbf{v}_{t}$ and $\mathbf{v}_{c}$ of the velocity screw of the probe. These components correspond to the force control, motion compensation and quality optimization tasks respectively. The force component $\mathbf{v}_{f}$ of the velocity screw, which is the task of highest priority, has already been defined in (3). Following the redundancy formalism described in [20], we define $\mathbf{P}_{f}$ and $\mathbf{P}_{f, t}$ the projection operators on the null-space of $\mathbf{L}_{f}$ and $\left(\mathbf{L}_{f}, \mathbf{L}_{t}\right)$ respectively:

$$
\begin{aligned}
\mathbf{P}_{f} & =\mathbf{I}_{6}-\mathbf{L}_{f}^{+} \mathbf{L}_{f} \\
\mathbf{P}_{f, t} & =\mathbf{P}_{f}-\mathbf{L}_{t}^{+} \mathbf{L}_{t},
\end{aligned}
$$

with $\mathbf{I}_{6}$ being the identity matrix of size 6 . Then, the velocity screw component corresponding to the motion compensation task is defined as

$$
\mathbf{v}_{t}=\left(\mathbf{L}_{t} \mathbf{P}_{f}\right)^{+}\left(\dot{e}_{t}^{*}-\mathbf{L}_{t} \mathbf{v}_{f}\right) .
$$

From (3), (4) and (8), we deduce $\mathbf{L}_{t} \mathbf{v}_{f}=0$ and $\mathbf{L}_{t} \mathbf{P}_{f}=\mathbf{L}_{t}$ (the tasks are decoupled), so that the vision control law can simply be written as

$$
\mathbf{v}_{t}=\mathbf{L}_{t}^{+} \dot{e}_{t}^{*}
$$

For the component of the velocity screw corresponding to the quality optimization task, the redundancy framework gives

$$
\mathbf{v}_{c}=\left(\mathbf{L}_{c} \mathbf{P}_{f, t}\right)^{+}\left(\dot{e}_{c}^{*}-\mathbf{L}_{c}\left(\mathbf{v}_{f}+\mathbf{v}_{t}\right)\right)
$$

and finally, the combined control law is obtained by summing the three components:

$$
\mathbf{v}=\mathbf{v}_{f}+\mathbf{v}_{t}+\mathbf{v}_{c} .
$$

We provide an illustration of the constraints induced by the different tasks in Fig 3. The force control task constrains one DOF, and it allows the probe to slide along the body's surface and to rotate around its imaging center. The target tracking task constrains the center of the target to be aligned with the central scan-line of the image, leaving one last DOF for confidence control. 


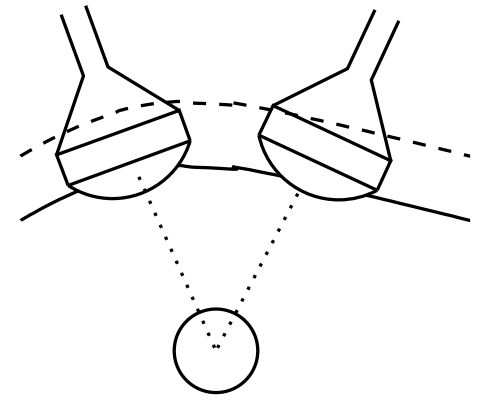

Fig. 3. Illustration of the constraints induced by the different control tasks. The plain line represents the body's surface, and the circle represents the target. The dashed line corresponds to the possible positions of the probe's center to obtain the desired contact force. The dotted lines illustrate the target tracking constraint.

\section{RESULTS}

\section{A. Experimental setup}

We use a 4DC7-3/40 convex ultrasound probe (Ultrasonix Medical Corporation, Canada), rigidly attached to the endeffector of a 6-axes Viper s650 robot (Adept Technology Inc., USA). The robot is equipped with a force/torque sensor. The prescan ultrasound frames are sent from the Ultrasonix system to a workstation (Intel Core i7, NVIDIA Quadro $\mathrm{K} 2000$ ), where our method is implemented. This workstation performs the different image processing steps required by our control framework in real-time:

- Conversion of the image from prescan $(128 \times 640)$ to postscan $(211 \times 152$, i.e., 2 millimeters per pixel $)$.

- Target tracking update in the postscan image.

- Downsampling of the prescan image to $64 \times 80$.

- Computation of the confidence map from the downsampled prescan image. This operation takes $9 \mathrm{~ms}$ on the downsampled image. On the full image, the computation would take $467 \mathrm{~ms}$, which would be too long for the control period.

- Conversion of the confidence map to postscan $(211 \times$ 152).

The tracking is based on an active contour model, from which we can extract the coordinates of the target's barycenter, as shown in Fig. 1(b). The desired control screw (13) is computed from the features extracted in the image processing steps, and applied to the robot. However, we limit the translational and rotational velocities to a maximum of $5 \mathrm{~mm} / \mathrm{s}$ and $5 \mathrm{deg} / \mathrm{s}$, respectively. The image processing is performed in less than $59 \mathrm{~ms}$, which is the period of the control loop. For the experiments, we use an ABDFAN ultrasound examination training model (Kyoto Kagaku Co., Japan), which resembles a human abdomen. Our experimental setup is illustrated in Fig. 4.

\section{B. Convergence}

We first present results related to the convergence of our system in a static environment. In these experiments, the phantom is not moving, and the probe is initially placed in such a position that the target is visible in the image. We
TABLE I

AVERAGE FEATURE ERRORS AFTER CONVERGENCE. THE RESULTS ARE DISPLAYED AS $\bar{e} \pm \sigma$, WHERE $\bar{e}$ IS THE MEAN VALUE OF THE SERIES, AND $\sigma$ IS THE STANDARD DEVIATION. COLUMN $e_{f}$ CORRESPONDS TO THE FORCE ERROR, $e_{t}$ TO THE TRACKING ERROR AND $e_{c}$ TO THE CONFIDENCE FEATURE ERROR.

\begin{tabular}{|c|c|c|c|}
\hline series & $e_{f}(\mathrm{~N})$ & $e_{t}(\mathrm{~mm})$ & $e_{c}(\mathrm{deg})$ \\
\hline 1 & $0.23 \pm 0.30$ & $0.30 \pm 0.26$ & $0.34 \pm 0.09$ \\
\hline 2 & $0.05 \pm 0.01$ & $0.35 \pm 0.13$ & $0.30 \pm 0.09$ \\
\hline 3 & $0.45 \pm 0.49$ & $0.51 \pm 0.24$ & $0.45 \pm 0.10$ \\
\hline Combined & $0.24 \pm 0.38$ & $0.39 \pm 0.22$ & $0.36 \pm 0.11$ \\
\hline
\end{tabular}

\section{TABLE II}

RELATIVE DIFFERENCE IN CONFIDENCE BETWEEN THE INITIAL STATE AND THE FINAL STATE. $\bar{c}$ IS THE MEAN VALUE OVER THE ENTIRE IMAGE. $\overline{c_{t}}$ IS THE MEAN VALUE OVER THE TARGET.

\begin{tabular}{|c|c|c|}
\hline experiment & $\bar{c}$ & $\overline{c t}$ \\
\hline 1 & $-10 \%$ & $+28 \%$ \\
\hline 2 & $+14 \%$ & $+88 \%$ \\
\hline 3 & $+3 \%$ & $+115 \%$ \\
\hline 4 & $+18 \%$ & $+105 \%$ \\
\hline 5 & $+16 \%$ & $+9 \%$ \\
\hline 6 & $-2 \%$ & $-14 \%$ \\
\hline 7 & $+26 \%$ & $+64 \%$ \\
\hline 8 & $-10 \%$ & $-18 \%$ \\
\hline 9 & $-12 \%$ & $+15 \%$ \\
\hline 10 & $-18 \%$ & $-20 \%$ \\
\hline 11 & $-11 \%$ & $+17 \%$ \\
\hline 12 & $+5 \%$ & $-8 \%$ \\
\hline 13 & $+12 \%$ & $-18 \%$ \\
\hline 14 & $+2 \%$ & $-24 \%$ \\
\hline 15 & $+10 \%$ & $-2 \%$ \\
\hline
\end{tabular}

conducted 15 experiments, with different initial configurations. The initial value of the feature errors ranged from 0.2 to $3.5 \mathrm{~N}$ for the force, 40 to $91 \mathrm{~mm}$ for the target's position and 6 to $16^{\circ}$ for the confidence feature. The experiments are separated in three series with different control gains:

1) $\frac{\lambda_{f}}{k}=0.001, \lambda_{t}=0.2$ and $\lambda_{c}=0.3$.

2) $\frac{\lambda_{f}}{k}=0.002, \lambda_{t}=0.4$ and $\lambda_{c}=0.4$.

3) $\frac{\lambda_{f}}{k}=0.002, \lambda_{t}=0.4$ and $\lambda_{c}=0.6$.

In each series, the desired contact force was set to $s_{f}^{*}=5 \mathrm{~N}$.

The desired behavior of the system with our control law is an exponential decrease of the feature errors. Therefore, we can expect the system to have converged after a period of $5 \tau$, where $\tau=\frac{1}{\lambda_{t}}$ is the characteristic time of the slowest task. We use this criterion to evaluate the precision of the convergence, by computing the mean feature error for $t>\tau$. We report in Table I the average final feature errors for each series of experiments. The evolution of the features during one of the experiments is presented in Fig. 5.

In addition to the feature errors, we registered the mean confidence of the image, as well as the mean confidence of the target. In order to estimate the impact of the control law on the confidence, we computed the relative difference of confidence between the initial state (namely, the $1^{\text {st }}$ second of the experiment) and the final state. The results are presented in Table II. In average, we observe an increase of the mean target confidence by $22 \%$. 


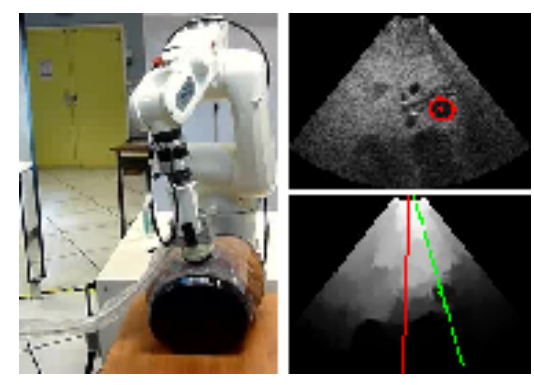

(a)

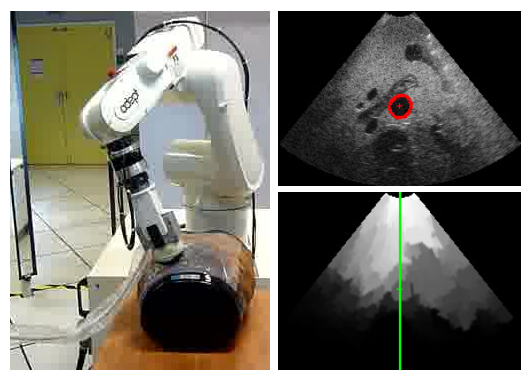

(b)
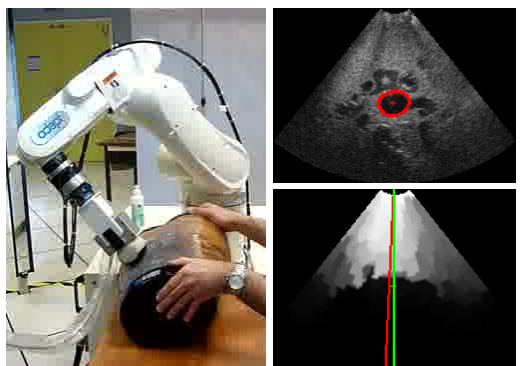

(c)

Fig. 4. Illustration of the experimental system, ultrasound image with the target, and confidence map with the angular feature. (a) Initial position. (b) After convergence. (c) During motion of the phantom. The red contour on the ultrasound images represents the boundary of the target. On the confidence maps, the red line represents the current configuration of the angular confidence feature, and the green line represents its desired configuration.

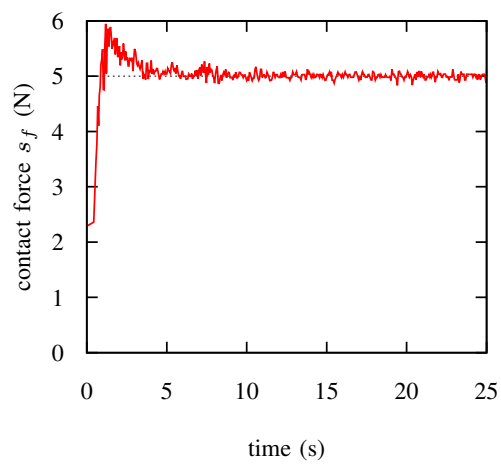

(a)

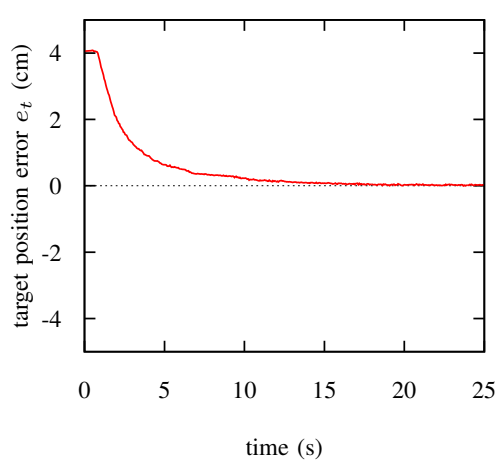

(b)

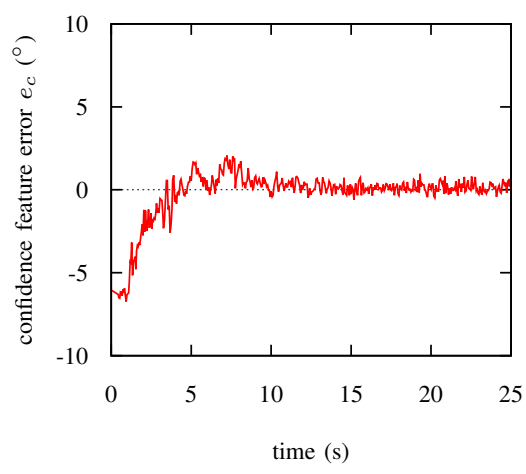

(c)

Fig. 5. Convergence: Evolution of the features during convergence in a static environment. (a) Contact force $s_{f}$ in N. (b) Target position error $e_{t}$ in $\mathrm{cm}$. (c) Confidence feature error $e_{c}$ in degrees.

\section{Motion compensation}

Finally, we present the results of an experiment demonstrating the use of our method for motion compensation. For this experiment, large motions were manually applied to the phantom, in order to test the reaction of the system in a nonstatic environment [see Fig. 4(c)]. We invite the reader to refer to the corresponding video, provided as supplementary material. The evolution of the different features during the experiment is presented in Fig. 6. In these plots, the gray parts represent the periods when the phantom was moved.

During the experiment, we applied three different motions to the phantom, interleaved with steady states:

- $13 \mathrm{~s}<t<24 \mathrm{~s}$ : fast rotation of the phantom towards the probe, generating a $14 \mathrm{~N}$ peak in the contact force, a displacement of $1 \mathrm{~cm}$ of the target in the image, and an error of 10 degrees for the confidence feature.

- $40 \mathrm{~s}<t<56 \mathrm{~s}$ : rotation back to the initial position, inducing a drop in the contact force.

- $67 \mathrm{~s}<t<92 \mathrm{~s}$ : slower rotation towards the probe, and fast release back to the initial position.

Each time, all three features converged back to their desired value during the steady state. During the transitional states, the feature errors remained within an acceptable range.

\section{DISCUSSION}

In this section, we discuss the prospects and limitations of our approach, as well as possibilities to overcome these limitations. The main point of our approach was to offer a simultaneous control of contact force, target position and image quality. The convergence analysis presented in Sect. III$\mathrm{B}$ shows that this control can be achieved in a decoupled manner. Namely, the quality optimization task did not disturb the force and target positioning tasks. Regarding the response time of the system, we presented results obtained with a control gain of up to 0.4 for the tracking and quality optimization tasks. This is sufficient to compensate for slow patient motion. To consider faster motions, it is necessary to increase the control gain. Currently, the bottleneck is the estimation of the confidence feature, which is not precise enough to have a stable control with a higher gain (see the oscillations of the feature error in Fig. 5(c) and Fig. 6(c)). In the current implementation, we compute the confidence map on a prescan image downsampled by 2 in width (number of scan lines) and 8 in height (depth). This downsampling was necessary in order to comply with the real-time requirement, as the computation of the confidence map was too long with the image at full resolution. However, it reduces the precision in the feature estimation, since (i) the estimation of the confidence map itself is less stable on a small image 


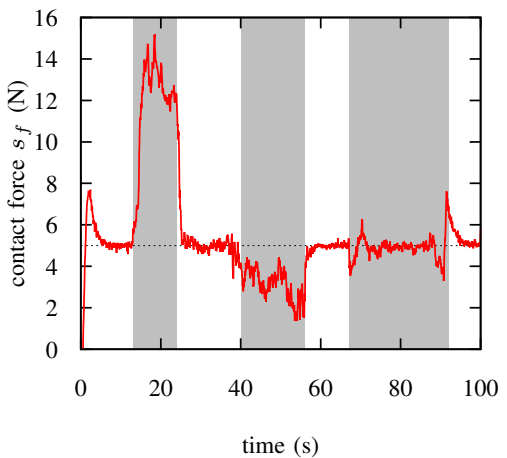

(a)

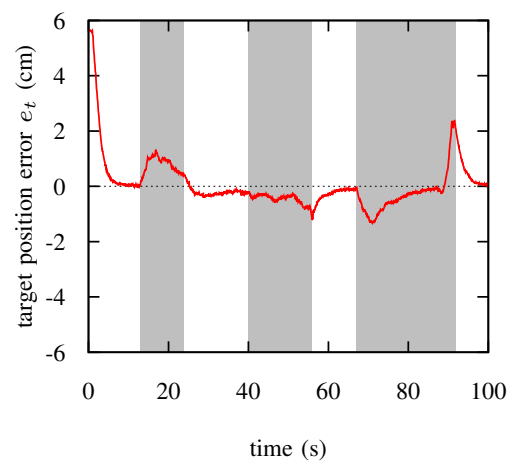

(b)

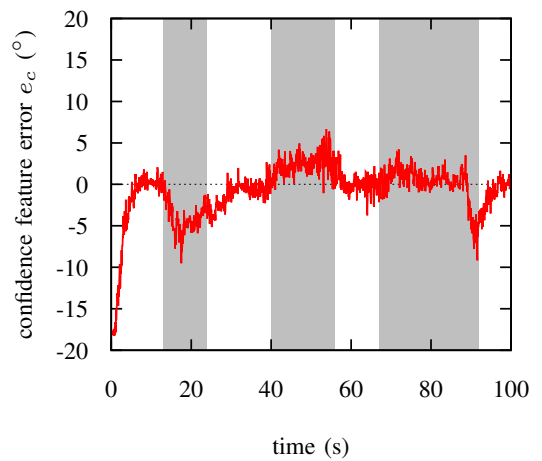

(c)

Fig. 6. Motion compensation: Evolution of the features during the motion compensation experiment. The gray parts ( 13 to $24 \mathrm{~s}$, 40 to $56 \mathrm{~s}$ and 67 to $92 \mathrm{~s}$ ) represent the periods during which the phantom was moved. (a) Contact force $s_{f}$ in N. (b) Target position error $e_{t}$ in cm. (c) Quality feature error $e_{c}$ in degrees.

and (ii) the precision of the confidence-based feature, defined in (5), depends on the resolution of the confidence map. One of our next steps will be to optimize the computation of the confidence map, which can be achieved such as proposed in [18]. With a more precise confidence-based feature, we expect to be able to increase the control gain of our system.

Another issue of interest is the out-of-plane control of the probe. We presented here a complete control of the in-plane motion (3 DOF). A 6-DOF control could be obtained with a 3D ultrasound probe using a similar control law, provided that we have a robust tracking of the target in $3 \mathrm{D}$.

\section{ACKNOWLEDGMENT}

This work was partially supported by the Bayerische Forschung Stiftung project RoBildOR.

\section{REFERENCES}

[1] B. B. Goldberg, J.-B. Liu, and F. Forsberg, "Ultrasound contrast agents: A review," Ultrasound Med. Biol., vol. 20, no. 4, pp. 319333, 1994.

[2] C. P. Loizou, C. S. Pattichis, M. Pantziaris, T. Tyllis, and A. Nicolaides, "Quality evaluation of ultrasound imaging in the carotid artery based on normalization and speckle reduction filtering," Med. Biological Eng. Comput., vol. 44, no. 5, pp. 414-426, 2006.

[3] S. Tsantis, S. Spiliopoulos, A. Skouroliakou, D. Karnabatidis, J. D. Hazle, and G. C. Kagadis, "Multiresolution edge detection using enhanced fuzzy c-means clustering for ultrasound image speckle reduction," Med. Phys., vol. 41, no. 7, p. 072903, 2014.

[4] S. H. C. Ortiz, T. Chiu, and M. D. Fox, "Ultrasound image enhancement: A review," Biomed. Signal Process. Control, vol. 7, no. 5, pp. 419-428, 2012.

[5] G. Kontaxakis, S. Walter, and G. Sakas, "EU-TeleInViVo: an integrated portable telemedicine workstation featuring acquisition, processing and transmission over low-bandwidth lines of 3D ultrasound volume images," in Proc. 2000 IEEE EMBS Int. Conf. Information Technology Applications in Biomedicine, pp. 158-163.

[6] D. De Cunha, P. Gravez, C. Leroy, E. Maillard, J. Jouan, P. Varley, M. Jones, M. Halliwell, D. Hawkes, P. Wells, et al., "The MIDSTEP system for ultrasound guided remote telesurgery," in Proc. Annu. Int. Conf. IEEE Engineering in Medicine and Biology Society, vol. 3, 1998, pp. 1266-1269.

[7] S. E. Salcudean, G. Bell, S. Bachmann, W.-H. Zhu, P. Abolmaesumi, and P. D. Lawrence, "Robot-assisted diagnostic ultrasound-design and feasibility experiments," in Medical Image Computing and ComputerAssisted Intervention, C. Taylor and A. Colchester, Eds. Springer Berlin Heidelberg, 1999, pp. 1062-1071.
[8] A. Vilchis, J. Troccaz, P. Cinquin, K. Masuda, and F. Pellissier, "A new robot architecture for tele-echography," IEEE Trans. Robot. Automat., vol. 19, no. 5, pp. 922-926, 2003.

[9] N. Koizumi, S. Warisawa, M. Nagoshi, H. Hashizume, and M. Mitsuishi, "Construction methodology for a remote ultrasound diagnostic system," IEEE Trans. Robot., vol. 25, no. 3, pp. 522-538, 2009.

[10] R. Nakadate, J. Solis, A. Takanishi, E. Minagawa, M. Sugawara, and K. Niki, "Out-of-plane visual servoing method for tracking the carotid artery with a robot-assisted ultrasound diagnostic system," in Proc. IEEE Int. Conf. Robotics and Automation, 2011, pp. 5267-5272.

[11] C. Nadeau and A. Krupa, "Intensity-based ultrasound visual servoing: modeling and validation with 2D and 3D probes," IEEE Trans. Robot., vol. 29, no. 4, pp. 1003-1015, 2013.

[12] A. Krupa, D. Folio, C. Novales, P. Vieyres, and T. Li, "Robotized teleechography: an assisting visibility tool to support expert diagnostic," IEEE Syst. J., 2014.

[13] I. Kuhlemann, R. Bruder, F. Ernst, and A. Schweikard, "WE-G-BRF09: Force- and image-adaptive strategies for robotised placement of 4D ultrasound probes," Med. Phys., vol. 41, no. 6, pp. 523-523, 2014.

[14] D. Kashyap Swathi Rangaraju, K. Kumar, and C. Renumadhavi, "Review paper on quantitative image quality assessment - Medical ultrasound images," Int. J. Eng. Res. Technol., vol. 1, no. 4, 2012.

[15] N. El-Zehiry, M. Yan, S. Good, T. Fang, S. K. Zhou, and L. Grady, "Learning the manifold of quality ultrasound acquisition," in Medical Image Computing and Computer-Assisted Intervention, K. Mori, I. Sakuma, Y. Sato, C. Barillot, and N. Navab, Eds. Springer Berlin Heidelberg, 2013, pp. 122-130.

[16] A. Karamalis, W. Wein, T. Klein, and N. Navab, "Ultrasound confidence maps using random walks," Med. image anal., vol. 16, no. 6, pp. 1101-1112, 2012.

[17] C. Schulte zu Berge, A. Kapoor, and N. Navab, "Orientation-driven ultrasound compounding using uncertainty information," in Information Processing in Computer-Assisted Interventions, D. Stoyanov, D. L. Collins, I. Sakuma, P. Abolmaesumi, and P. Jannin, Eds. Springer International Publishing, 2014, pp. 236-245.

[18] C. Schulte zu Berge, D. Declara, C. Hennersperger, M. Baust, and N. Navab, "Real-time uncertainty visualization for B-mode ultrasound," in Proc. Scientific Visualization, 2015.

[19] P. Chatelain, A. Krupa, and N. Navab, "Optimization of ultrasound image quality via visual servoing," in Proc. IEEE Int. Conf. Robotics and Automation, 2015, pp. 5997-6002.

[20] B. Siciliano and J.-J. Slotine, "A general framework for managing multiple tasks in highly redundant robotic systems," in IEEE Int. Conf. Advanced Robotics, 1991, pp. 1211-1216.

[21] L. Grady, "Random walks for image segmentation," IEEE Trans. Pattern Anal. Machine Intell., vol. 28, no. 11, pp. 1768-1783, 2006.

[22] T. Li, A. Krupa, and C. Collewet, "A robust parametric active contour based on fourier descriptors," in IEEE Int. Conf. Image Processing, 2011, pp. 1037-1040. 